\title{
Liquefaction processes and characterization of liquefied products from waste woody materials in different acidic catalysts
}

\author{
Q. Wang ${ }^{1}$, Q. Chen ${ }^{1}$, P. Apaer ${ }^{1}$, N. Kashiwagi ${ }^{1}$, H. Kurokawa ${ }^{1}$, \\ K. Sugiyama ${ }^{2}$ X. Wang ${ }^{3} \&$ X. Guo ${ }^{4}$ \\ ${ }^{I}$ Department of Environmental Science and Technology, \\ Graduate School of Science and Engineering, Saitama University, Japan \\ ${ }^{2}$ Hachinohe National College of Technology, Japan \\ ${ }^{3}$ Center for Environmental Science in Saitama, Japan \\ ${ }^{4}$ School of Environmental and Chemical Engineering, \\ Shanghai University, China
}

\begin{abstract}
The liquefaction process is one of the promising techniques for effective utilization of woody biomass, for the lignocelluloses can be converted to liquid reactive material, as eco-polymeric materials. Japanese cedar (Cryptomeria Japonica), as an abundant waste softwood material, was selected and used in our wood liquefaction experiment. In order to investigate the basic characteristics and potentially harmful metal contents, the composition and metal elements of waste woody samples had been determined, and based on the methods of Japanese Industrial Standard (JIS) and by an ICP-AES, separately. Then the waste woody samples were liquefied by a phenol wood liquefaction according to the orthogonal test $\mathrm{L}_{9}\left(3^{4}\right)$, in order to obtain relatively less residue by different reaction conditions. It is thought that sulfuric acid plays an important role in retarding the condensation reaction during the acid-catalyzed phenol liquefaction because of the dehydration, and it can be summarized that the most influential factors of the wood liquefaction conditions were obtained within the setting ranges on four factors and three levels by using the orthogonal tests. In the acidic catalyst comparison experiment, as a result, when using concentrated sulfuric acid as the strong acidic catalyst, the minimum of residual content had reached $9.71 \%$. According to these experimental results, the new liquefied samples
\end{abstract}


demonstrated the relationships between some characteristics of liquefied products from waste woody materials through analyses of Japanese phenolic resin industry testing series such as viscosity, nonvolatility and so on. The results showed that whether the viscosity or novolatility the greatest changed of the liquefied materials had taken place in reaction time during the 6 hours. In addition, in order to find some structural changes and to clarify the mechanism of respective products, the analysis by combined phenol and free phenol was carried out in our further studies.

Keywords: waste woody materials, liquefaction, phenol, liquefied products, orthogonal test, viscosity, acidic catalysts.

\section{Introduction}

In China and Japan it is estimated that more than a million tones of timber wastes is generated each year [1]. Construction woody wastes have become the major source of solid organic wastes [2]. Waste management is becoming more important against the alarming warning signals in industry and to people's lives. Reuse and recycling to reduce the wastes are considered as the only methods to recover those wastes, such as timber waste. On the other hand, the polymeric material can be far more seriously damaging in the natural environment, because it is difficult to be degraded in a short period of its life time [3]. The technologies of liquefaction can convent biomass resources into eco-polymeric materials which can replace the pre-polymer which is made from oil resource [4], and solvent liquefaction is one of the effective methods as a solution to the problem [5]. The acid catalyst can degrade the woody components to small ingredients, and then these ingredients react with phenol to form a derivative and dissolve in the liquefaction solvent.

Lin et al. used GG guaiacylglycerol- $\beta$-guaiacyl ether, as a lignin model compound, carried out the relevant research about liquefaction reaction mechanism of lignin compounds in the presence of sulfuric acidic catalyst $[6,7]$ and without catalysts $[8,9]$. They had also attempted to clarify the reaction mechanism of cellulose with phenol under the acid-catalyzed conditions. It was found that the yield ratio of various compounds in the end reaction product is greatly dependent on the reaction conditions, i.e. phenol/cellobiose ratio, concentration of acidic catalyst, reaction temperature, and reaction time. Therefore, by controlling these reaction conditions, it is possible to adjust the structure and properties of the end liquefaction products [10]. Yamada et al. [11] reported that the acid catalyzed liquefaction of cellulose with polyhydric alcohols includes complicated reaction where cellulose is decomposed to glucose, the glucose transforms to 5-hydroxymethylfurfural. However, Ono et al. [12] reported a possible chemistry of phenol assisted liquefaction: cellulose is degraded to cello-oligosaccharides and glucose by sulfuric acidic catalyst, then its pyranose ring structure is subject to decomposition and then recombination of its decomposed fragments and phenols would occur in the process of phenol liquefaction. Zhang et al. [13] researched the liquefaction mechanism of cellulose in phenol. They indicated the pyranose that decomposed from cellulose 
could combine with phenol to form a hydroxyl benzyl form derivative. Lee and Ohkata [14] indicated wood could be rapidly liquefied at the supercritical temperature. Under this condition, over $90 \%$ of wood was liquefied within 0.5 min, as the new liquefaction methods.

In our study, we found that reaction time is the most important factor in the process of liquefaction according to the result of orthogonal tests, in spite of acidic catalysts used as another important factor. Especially, the extremely rapid and complex changes had taken place during the first 2 hours. A better understanding of liquefaction process and characterization of liquefied products should be informed through a series of experiments and analysis. In addition, the heavy metal elements such as $\mathrm{Cr}, \mathrm{Cu}$ and $\mathrm{Pb}$ which sourced from chemical preservatives had been tested for the environmental impact assessment, because the liquefaction reaction carried out in strong acidic conditions.

\section{Materials and methods}

\subsection{Materials and different acidic catalysts}

The Japanese cedar (Cryptomeria Japonica), as the waste woody materials of the thin woody materials and construction woody materials, had been collected from the campus of Saitama University and waste wood processing factories in Kanto region of Japan. The air-dried samples were milled in smashing equipment and retained at the size ranges of 10-100 mesh screens were used for the test. All the flour samples of woody materials were dehydrated in an oven at $107^{\circ} \mathrm{C}$ for 24 hours. The main different acidic catalysts such as $\mathrm{H}_{2} \mathrm{SO}_{4}(95 \%), \mathrm{H}_{3} \mathrm{PO}_{4}(85 \%)$, phenol and other reagents were prepared with analytical grade reagents in accordance with the Japanese industrial standard (JIS) supplied by Wako Pure Chemicals, Co. Ltd., Japan.

\subsection{Chemical composition of liquefied woody materials}

The composition analysis of liquefied woody material had divided into three parts. In the industry analysis, the test of ash which is the residue powder left after the combustion of wood is performed according to JIS-M8812. The moisture content of wood is determined by measuring the weight loss after drying the sample at $105^{\circ} \mathrm{C}$ in an oven. Moisture content is determined on a separate portion of the sample not used for the other analyses. Volatile matter is tested by heating the wood sample to $900^{\circ} \mathrm{C}$ under carefully controlled conditions and measuring the weight loss. The fixed-carbon contents in woody materials were determined by subtracting the percentages of moisture, volatile matter, and ash from each sample. The second part of analysis was elemental analysis which according to JIS-M8813 by the CHN coder (Model MT-5, Yanaco Co. Ltd., Japan). At the part of component quantitative analysis, wood extraction procedures in $1 \% \mathrm{NaOH}$ extract most extraneous components, some lignin, and low molecular weight hemicelluloses and degraded cellulose. The solubility of woody material in EtOH/benzene (benzene is a known carcinogen; 
toluene can be substituted) in a 1:2 volume ratio will give a measure of the extractives content. The wood meal is refluxed 6 hours in a soxhlet flask, and the weight loss of the extracted, dried woody material is measured. Holocellulose is the total polysaccharide (cellulose and hemicelluloses) contents of woody material, and methods for its determination seek to remove all of the lignin from woody material without disturbing the carbohydrates. Alpha cellulose is obtained after treatment of the holocellulose with $17.5 \% \mathrm{NaOH}$. Cross and bevan cellulose consists largely of pure cellulose, but also contains some hemicelluloses. It was obtained by chlorination of wood meal, followed by washing with $3 \% \mathrm{SO}_{2}$ and $2 \%$ Sodium Sulfite. The lignin contents of waste woody materials presented in the tables are Klason lignin, the residue remaining after solubilizing the carbohydrate with strong mineral acid. The usual procedure was to treat a shorter method treats the sample with $72 \% \mathrm{H}_{2} \mathrm{SO}_{4}$ at $30^{\circ} \mathrm{C}$ for one hour, followed by an hour at $120^{\circ} \mathrm{C}$ in $3 \% \mathrm{H}_{2} \mathrm{SO}_{4}$. The analytical results of the waste woody materials used in our experiments were summarized in table 1 and table 2 .

Table 1: $\quad$ Industrial and elemental analysis (wt. \%) of waste woody materials.

\begin{tabular}{cccc|cccc}
\hline \multicolumn{3}{c}{ Analysis of waste woody materials } & \multicolumn{5}{c}{ Elements analysis } \\
\hline Ash & VM & M & FC & C & H & N & O \\
0.6 & 79.1 & 7.7 & 12.6 & 48.9 & 6.1 & 0.4 & 44.6 \\
\hline
\end{tabular}

VM: Volatile Matter, M: Moisture, FC: Fixed Carbon.

Table 2: $\quad$ Composition analysis (wt. \%) of waste woody materials.

\begin{tabular}{lcc}
\hline & Composition analysis & $\begin{array}{c}\text { Liquefied woody } \\
\text { materials }\end{array}$ \\
\hline \multirow{3}{*}{ Carbohydrate } & Holocellulose $^{\mathrm{a}}$ & 55 \\
& Cross and Bevan Cellulose & 43 \\
& Alpha Cellulose $^{\mathrm{c}}$ & 29 \\
Slason Lignin & 27 \\
\hline \multirow{2}{*}{ Solubility } & $1 \% \mathrm{NaOH}$ & 12.81 \\
& EtOH/Benzene & 3.25 \\
\hline
\end{tabular}

${ }^{a}$ Holocellulose is the total carbohydrate content of waste woody materials.

${ }^{b}$ Cross and Bevan Cellulose is largely pure cellulose but contains some hemicelluloses.

${ }^{c}$ Alpha Cellulose is nearly pure cellulose.

\subsection{Heavy metal analysis in thin and construction woody materials by an ICP-AES}

The heavy metals such as $\mathrm{Cr}, \mathrm{Cu}$, and $\mathrm{Pb}$ which are sourced from wood material had been tested, because the liquefaction reaction is carried out in strong acidic conditions. The inductively coupled plasma atomic emission spectroscopy (ICPAES Optima 5300 DV, Perkin Elmer Co., Ltd., Japan) analysis had been using as the analysis project, the two kinds of woody material were digested with concentrated $\mathrm{HNO}_{3}(3 \mathrm{ml})$ and $\mathrm{HF}(3 \mathrm{ml})$, then the solutions were heated to 
dryness on an aluminum heating block at $100^{\circ} \mathrm{C}$. After $1 \mathrm{~h}$ adding $\mathrm{HNO}_{3}(10 \mathrm{ml})$ and HF $(2 \mathrm{ml})$, these extract solutions were left overnight on a heating block, and the temperature raised slowly to $200^{\circ} \mathrm{C}$ and heated to dryness. The residue in the tubes was then leached with $0.1 \mathrm{~N}$ of $\mathrm{HNO}_{3}$, and made-up to final volumes of 50 $\mathrm{ml}$ sample to analysis the heavy metal ions.

\subsection{The orthogonal test $\mathrm{L}_{9}\left(3^{4}\right)$ using in the liquefaction of woody materials}

An orthogonal test $\mathrm{L}_{9}\left(3^{4}\right)$ design was used for optimization the extraction conditions of liquefaction of waste woody materials in the present sulfuric acidic catalysts. In this study, the setting conditions were carried out at reaction temperatures of $140^{\circ} \mathrm{C}, 150^{\circ} \mathrm{C}$ and $160^{\circ} \mathrm{C}$, the ratio of phenol and air-dried waste woody materials were 1:3.0, 1:3.5 and 1:4.0, extraction time 1.5,2, 2.5 and additive weight of catalysts 6,8 and 10 (wt.\% of solvent) respectively on the basis of the single-factor test. Table 3 shows the experimental conditions for the extraction of liquefaction. It is according to the other experimental conditions which are usually used in the wood liquefaction experimental. We considered that high temperature will affect the volatile of phenol, and the lower temperature will affect the speed of experimental liquefaction. Other experimental factor will follow interrelated factor such as selected liquefaction temperature. In this orthogonal test, we hope to found out the better factors in these factors and the optimal conditions in setting conditions.

Table 3: $\quad$ Experiment factors and levels for orthogonal test.

\begin{tabular}{lccc}
\hline \multicolumn{1}{c}{ Variable } & Level 1 & Level 2 & Level 3 \\
\hline A: Extraction temperature $\left({ }^{\circ} \mathrm{C}\right)$ & 140 & 150 & 160 \\
B: Extraction time (hour) & 1.5 & 2.0 & 2.5 \\
C: Ratio of phenol and air-dry wood & $1: 3.0$ & $1: 3.5$ & $1: 4.0$ \\
D: Additive weight of catalysts (wt $\%)$ & 6 & 8 & 10 \\
\hline
\end{tabular}

\subsection{Characteristics of phenol liquefaction of woody materials}

\subsubsection{Comparison experimental of different acidic catalysts}

Experimental liquefaction of waste woody materials were carried out by varying the weight charge ratio of wood to phenol with $1 / 4$ and the reaction temperature at $150^{\circ} \mathrm{C}$ by oil bath. Into a $500 \mathrm{ml}$ three necked flask equipped with stirrer. In addition, the same weight ratio of wood/phenol/ $\mathrm{H}_{2} \mathrm{SO}_{4}, \mathrm{HCl}$ and $\mathrm{H}_{3} \mathrm{PO}_{4}$ were used in the comparison experimental. The content was heated in 2 hours.

Otherwise, the characteristics of produce research experimental was liquefied for 6 hours with two kinds of acidic catalysts; we tested the residues, viscosity, nonvolatility, combined phenol and free phenol at every hour to evaluate the correlation.

\subsubsection{Measurement of liquefaction residue}

The liquefied woody material was weighted and diluted with methanol, and Whatman filter paper was used to collect the liquefied residue. The liquefied 
residue was dried in a heating oven at $105^{\circ} \mathrm{C}$ in 24 hours and calculated the residues by equation (1).

$$
\text { Liquefied residue }(w t \%)=(\text { Residue } / \text { Raw material }) \times 100 \%
$$

\subsubsection{Viscosity measurement of liquefied waste woody materials}

The viscosity of liquefied woody materials was measured with a Viscometer (Model VT-04F, RION Co. Ltd., Japan) by each hour within 6 hours in 300ml standard beaker at $25 \pm 3^{\circ} \mathrm{C}$. The viscosity is measured using a special mechanism to obtain direct readings in decipascal-seconds (dPa.s) (accuracy: within $\pm 10 \%$ of meter indication, rotating velocity of the rotor: $62.5 \mathrm{rpm})$.

\subsubsection{Nonvolatility in the liquefied waste woody materials}

About (1.5-3.0) $\pm 0.5 \mathrm{~g}$ of liquefied waste woody materials were weighted and dried using a oven at $180 \pm 1^{\circ} \mathrm{C}$ by 1 hour according to JIS-K6910 to remove the un-reacted phenol and nonvolatility in the liquefied waste woody materials can be calculated by the following equation (2).

$$
\text { Nonvolatility }(w t \%)=(\text { Residue after oven-dry } / \text { The sample }) \times 100 \%
$$

\subsubsection{Combined phenol and free phenol in the liquefied woody materials}

The combined phenol and free phenol were used to show the phenol existing in the liquefied waste woody materials that were combined with wood components or free in the system [5], respectively. Both the combined and free phenol was calculated from the nonvolatile content in the liquefied wood. The combined phenol was calculated by subtracting the content of wood used in the liquefaction system from the nonvolatile content after liquefaction. The free phenol (unbound phenol) was calculated by subtracting the content of combined phenol from the content of phenol used in the initial liquefaction system. The combined phenol and free phenol can be expressed as the following equation (3) and equation (4), respectively.

$$
\begin{gathered}
\text { Combined phenol }(w t \%)=[\text { Nonvolatility }-w /(w+p+c)] \times 100 \% \\
\text { Free phenol }(w t \%)=[p /(w+p+c)-\text { Combined phenol }] \times 100 \%
\end{gathered}
$$

Here, $w$ : weight of woody material, $p$ : phenol weight, and $c$ : catalyst weight.

\section{Results and discussions}

\subsection{Comparison of heavy metal analysis between thin woody material and construction woody material (Liquefied woody materials)}

Analytical results of the heavy metal concentrations in the thin woody materials and construction woody materials are showed in figure 1 by ICP-AES analysis. It shows that the arithmetic means of $\mathrm{Co}, \mathrm{Cr}, \mathrm{Cu}, \mathrm{Ni}$ and $\mathrm{Pb}$ are $0.02,0.04,0.14$, 0.04 and $0.09 \mathrm{mg} \mathrm{g}^{-1}$ of sample, respectively, both of woody material have the nearly concentrations of these heavy metals. These metals are a cause of environmental pollution, meanwhile, an amount of concentrated acidic catalyst 
was used in the liquefaction process, the reaction between the catalyst and heavy metals, which may come from wood preservatives, was considered in the liquefaction system.

Klok and de Roos [15] showed that heavy metal contamination can induce two major effects on the ecosystem level: (a) accumulation of e.g. Cr can lead to risks of secondary poisoning, while (b) organism disappears even at low levels of $\mathrm{Cu}$ in soil. The conditions would occurred if the product of liquefied woody materials should been landfilled in soil. In order to further understand the state of these metals after liquefaction, ion-exchange test will be carrying out to find whether there harmful ions $\left(\mathrm{Pb}^{2+}, \mathrm{Cd}^{2+}\right.$ and $\left.\mathrm{Ni}^{2+}\right)$ would be produced.

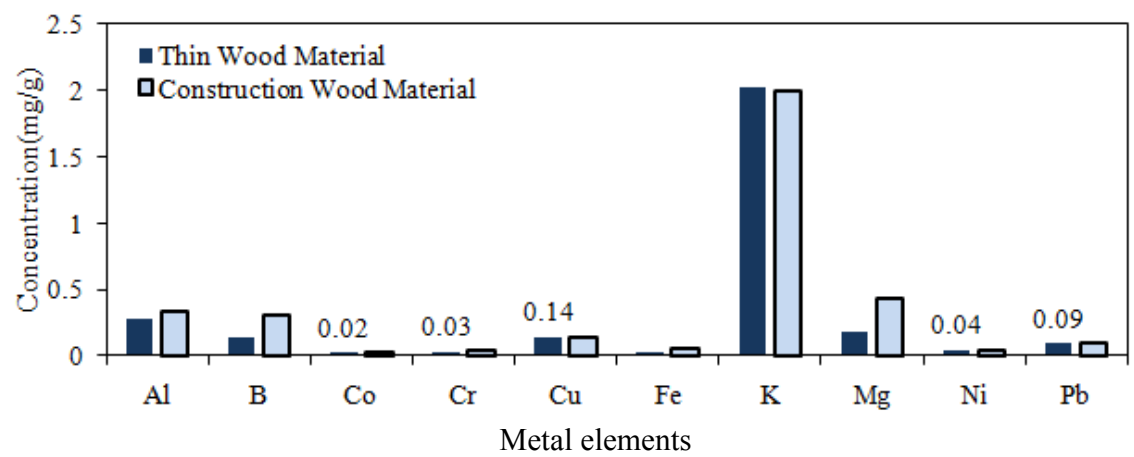

Figure 1: $\quad$ Some metal elements in thin and construction woody materials.

\subsection{Influential factors of liquefaction examined by the orthogonal tests}

In our study, all the influential factors were examined using an orthogonal $\mathrm{L}_{9}\left(3^{4}\right)$ test design. The total evaluation indexes were used to analysis by the statistical method. The results of orthogonal test and extreme difference analysis were presented in table 4.

The liquefied residue from each test in the liquefied products was weighted. As shown in table 4, we can find that the influence to the mean liquefied residue of the compounds decreases in the order: $\mathrm{B}$ (extraction time) $>\mathrm{A}$ (extraction temperature) $>\mathrm{C}$ (ratio of phenol and wood) $>\mathrm{D}$ (catalyst weight) according to compare the $\mathrm{R}$ values.

The extraction time was found to be the most important factor of the yield. In other words, the minimum residue of the liquefied products can be obtained when extraction time, extraction temperature, ratio of phenol and wood, catalyst weight were 2 hour, $150^{\circ} \mathrm{C}, 1: 4$ and $8 \%$, respectively. However, we cannot get the best extraction conditions only based on these out comes in table 4 . Although it can be summarized that extraction time are the most influential factors within the wood liquefaction conditions within the setting ranges on four factors and three levels by using the orthogonal tests. The tangible results provided us with the correct catalyst using in the orthogonal tests, and it should be in the comparison some characteristics of the products in the further experiments. 
Table 4: $\quad$ The scheme and analytical values of $\mathrm{L}_{9}\left(3^{4}\right)$ orthogonal test.

\begin{tabular}{cccccc}
\hline $\begin{array}{c}\text { Sample } \\
\text { No. }\end{array}$ & $\begin{array}{c}\text { A: extraction } \\
\text { temperature } \\
\left({ }^{\circ} \mathrm{C}\right)\end{array}$ & $\begin{array}{c}\text { B:extraction } \\
\text { time } \\
(\mathrm{hr})\end{array}$ & $\begin{array}{c}\text { C: ratio of } \\
\text { phenol and } \\
\text { wood }\end{array}$ & $\begin{array}{c}\text { D: catalyst } \\
\text { weight } \\
\text { (wt.\%) }\end{array}$ & $\begin{array}{c}\text { Liquefied } \\
\text { residue } \\
\text { (wt.\%) }\end{array}$ \\
\hline 1 & 140 & 1.5 & $1: 3.0$ & 6 & 17.49 \\
2 & 140 & 2.0 & $1: 3.5$ & 8 & 10.83 \\
3 & 140 & 2.5 & $1: 4.0$ & 10 & 10.27 \\
4 & 150 & 1.5 & $1: 3.5$ & 10 & 14.28 \\
5 & 150 & 2.0 & $1: 4.0$ & 6 & 11.20 \\
6 & 150 & 2.5 & $1: 3.0$ & 8 & 11.83 \\
7 & 160 & 1.5 & $1: 4.0$ & 8 & 9.27 \\
8 & 160 & 2.0 & $1: 3.0$ & 10 & 9.87 \\
9 & 160 & 2.5 & $1: 3.5$ & 6 & 10.48 \\
$K_{1}$ & 38.59 & 41.04 & 39.19 & 39.17 & \\
$K_{2}$ & 37.31 & 31.90 & 35.59 & 31.93 & \\
$K_{3}$ & 29.62 & 32.58 & 30.74 & 34.42 & \\
$R$ & $8.97^{-5}$ & 9.14 & 8.45 & 7.24 & \\
\hline
\end{tabular}

${ }^{\mathrm{a}} K_{i}^{n}=\sum n_{i}(n=A, B, C, D ; i=$ Level 1, 2, 3) in dashed line.

${ }^{\mathrm{b}} R=\max \left\{K_{i}^{n}\right\}-\min \left\{K_{i}^{n}\right\}(i=1,2,3)$.

\subsection{Comparison some characteristics of phenol liquefaction of wood}

We have chosen three kinds of catalyst in the liquefaction experiments to determine the difference of liquefaction process. In figure 2 which can found that through the use of a concentration of $95 \%$ sulfuric acid, the reaction use $10 \%$ of the total weight of the reactants, the residual substance at least to 9.71 percent.

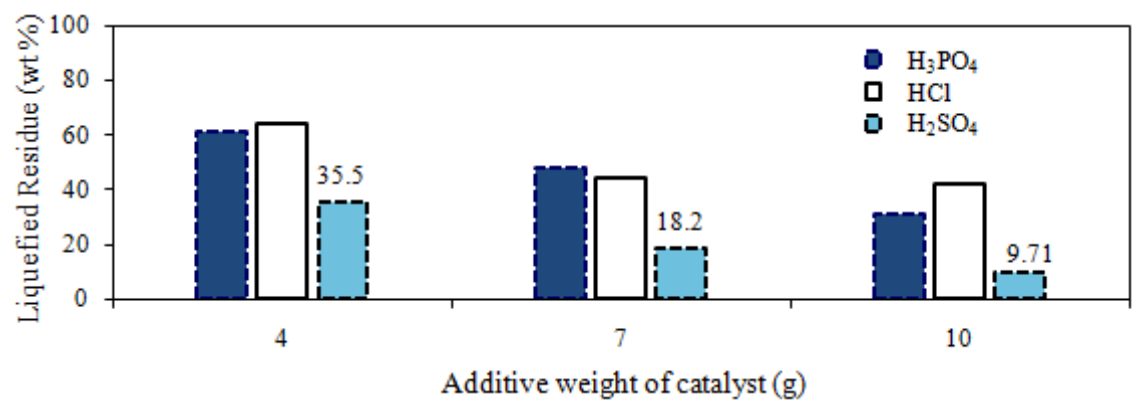

Figure 2: Results of different concentrations of the different acidic catalysts in the liquefaction experiments.

Liquefaction catalyst with hydrochloric acid, the residue rate had increased with the amount of catalyst is decreasing. However, due to hydrochloric acid is highly volatile and the liquefaction test was carried out under atmospheric pressure, hydrochloric acid at high temperature under the action of hydrogen chloride gas in the form of evaporation [16]. The product residue rate of $40 \%$ is 
relatively higher than phosphoric acid and sulfuric acid. When using phosphoric acid as catalyst, the results showed that at the same temperature conditions, the use of phosphoric acidic catalyst on the liquefaction of wood there was significant effects of residue increased with the increase in the amount of catalyst decreases. However, when the catalyst dosage is greater than $7 \%$, then the extent of catalytic has declined.

\subsection{Characteristics of phenol liquefaction of woody materials with two different acidic catalysts}

In order to understand the characteristics of produce in different catalysts, we taken liquefaction experiment for 6 hours with two kinds of catalysts, we tested the residue, viscosity, nonvolatility, combined phenol and free phenol at every hour to evaluate the correlation. The results are shown in table 4, figure 3 and figure 4. Figure 3 shows the properties of phenol liquefied Japanese cedar. The liquefied wood that used $\mathrm{H}_{2} \mathrm{SO}_{4}$ as an acidic catalyst had the unliquefied wood residue lower than that used $\mathrm{H}_{3} \mathrm{PO}_{4}$ as an acidic catalyst. This result indicated Japanese cedar liquefied with $\mathrm{H}_{2} \mathrm{SO}_{4}$ as a catalyst had a better liquefaction reaction than $\mathrm{H}_{3} \mathrm{PO}_{4}$. Nevertheless the former had viscosity (950-1000 dPa.s) higher than the latter (90-100 dPa.s) in table 4.

Table 5: Viscosity of liquefied products that used $\mathrm{H}_{2} \mathrm{SO}_{4}$ and $\mathrm{H}_{3} \mathrm{PO}_{4}$ catalysts in 6 hours.

\begin{tabular}{ccccccc}
\hline Time (hour) & 1 & 2 & 3 & 4 & 5 & 6 \\
\hline Viscosity of produce in $^{\text {a }}$ & $20-$ & $135-$ & $250-$ & $440-$ & $600-$ & $950-$ \\
$\mathrm{H}_{2} \mathrm{SO}_{4}{ }^{\text {a }}$ (dPa.s) & 23 & 140 & 260 & 460 & 650 & 1000 \\
$\begin{array}{c}\text { Viscosity of produce in } \\
\mathrm{H}_{3} \mathrm{PO}_{4}{ }^{\text {b }} \text { (dPa.s) }\end{array}$ & $8-9$ & $23-25$ & $35-37$ & $60-65$ & $85-90$ & $90-100$ \\
\hline
\end{tabular}

${ }^{a}$ The weight ratio of phenol/wood/ $\mathrm{H}_{2} \mathrm{SO}_{4}$ used for liquefaction was 4/1/0.08.

${ }^{b}$ The weight ratio of phenol/wood/ $\mathrm{H}_{3} \mathrm{PO}_{4}$ used for liquefaction was 4/1/0.19.

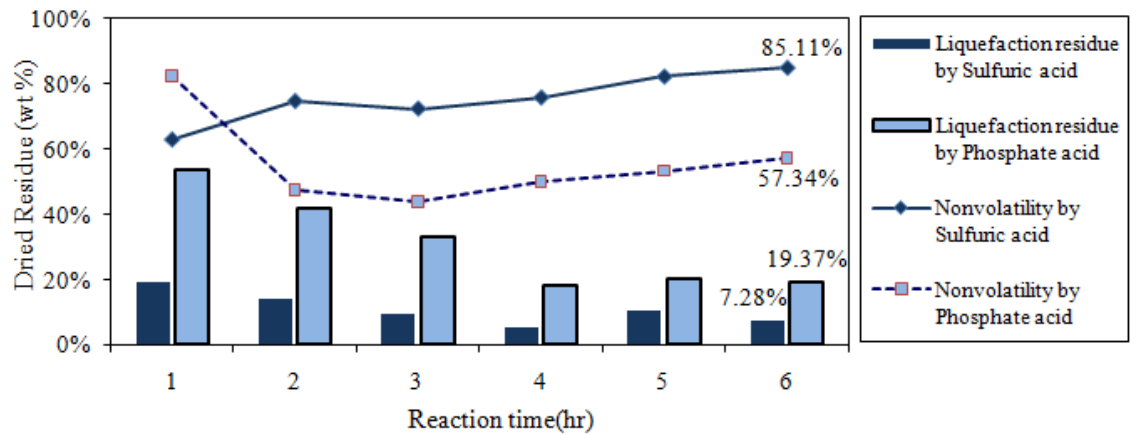

Figure 3: Relationship between liquefaction residues and nonvolatility in liquefied products that used $\mathrm{H}_{2} \mathrm{SO}_{4}$ and $\mathrm{H}_{3} \mathrm{PO}_{4}$ in 6 hours (wt.\%). 


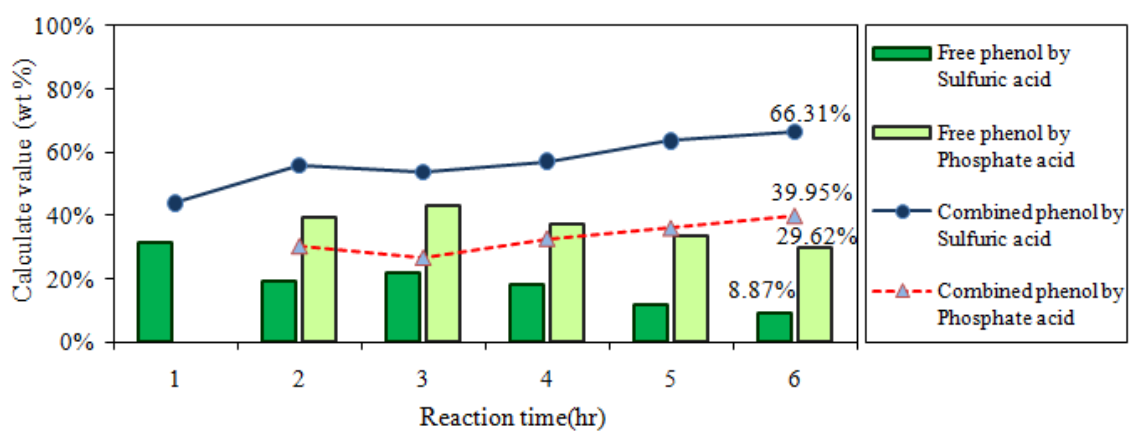

Figure 4: Calculate values of combined phenol and free phenol that used $\mathrm{H}_{2} \mathrm{SO}_{4}$ and $\mathrm{H}_{3} \mathrm{PO}_{4}$ in 6 hours (wt.\%).

The value would be nearly 10 times higher which had showed that the reaction time in 6 hours. The large amount of $\mathrm{H}_{3} \mathrm{PO}_{4}$ catalyst had been used in the experimental liquefaction shown in figure 3.

From these results, we can also find that the greatest changes in the viscosity of liquefied products had taken place in reaction time during the 6 hours which used $\mathrm{H}_{2} \mathrm{SO}_{4}$. However, it has small changes in the viscosity of liquefied products using $\mathrm{H}_{3} \mathrm{PO}_{4}$ as the acidic catalyst. During the first 3 hours, the effect of the acidic catalyst is not very clear in $\mathrm{H}_{3} \mathrm{PO}_{4}$ which could be understood from the results given in figure 3 .

After liquefaction, the nonvolatile contents in the liquefied products were $85.11 \%$ for that used $\mathrm{H}_{2} \mathrm{SO}_{4}$ in the six hour and $57.34 \%$ that used $\mathrm{H}_{3} \mathrm{PO}_{4}$. These higher values of nonvolatility might be caused by some of the phenol that would combine with the wood component to form some derivatives [5].

From our experiments, we can also summarize the nonvolatility value has increased with time for $\mathrm{H}_{3} \mathrm{PO}_{4}$, increase with time when used $\mathrm{H}_{2} \mathrm{SO}_{4}$ as a catalyst. However, we have not calculated the values of combined phenol and free phenol at the first hour for $\mathrm{H}_{3} \mathrm{PO}_{4}$, because it also remains many residues after liquefaction which may be unliquefied woody material in the original samples. Zhang et al. [13] indicated the cellulose would be degraded to oligosaccharide and glucose in phenol with an acidic catalyst under the high temperature. The pyranose ring would decompose further and combine with phenol to form their derivatives. The derivatives formed had the hydroxyl benzyl group but not the phenoxyl-form. Because the phenolic functional group that kept in the derivatives had the similar reactivity as phenol. In our study, the combined phenol with $\mathrm{H}_{2} \mathrm{SO}_{4}$ is better than used $\mathrm{H}_{3} \mathrm{PO}_{4}$, because the more free phenol will be residues in the liquefied products after liquefaction.

\section{Conclusion}

We investigated the heavy metal concentrations in the thin woody materials and construction woody materials. The analytical results have shown us that it had 
some heavy metals contained in wood such as $\mathrm{Co}, \mathrm{Cr}, \mathrm{Cu}, \mathrm{Ni}$ and $\mathrm{Pb}$. The possible resources of these metals may come from preservatives, paint or other chemicals which were used in wood processing. Meanwhile, now a common way of waste disposal is landfill, so it is necessary to be considered that the concentrations of heavy metals would take the reaction with the various catalysts, and the concentrations should be determined before liquefaction. Otherwise, as the new material products, the standard amounts of metals contained should be established before use in order to avoid the soil contamination when it would be landfill in the soil.

The results of orthogonal test tell us that the maximum impact factors can be obtained in the setting range value on four factors and three levels. Thus, it is concluded that if we want to find the maximum or minimum environmental impact factors in liquefaction process by the orthogonal test. However, in this study, we cannot get the best extraction conditions by the orthogonal test, because some other impact factors such as stirring speed will also affect the results. Therefore, we cannot recommend that in order to seek the best conditions by only orthogonal test. On other words, the minimum residual value in liquefied products is what we want, and the orthogonal test can help us to demand and choose better factors in less experiments.

The effect of sulfuric acid as the acidic catalyst is obviously better than phosphoric acid, which could be known by some properties of viscosity, nonvolatility, combined phenol and free phenol. This may be due to the role of sulfuric acid is a strong acid, but we need to analyze the performance of the liquefied products in our further studies. In other words, we cannot say this kind of acidic catalysts is most suitable for the liquefaction of woody materials, the performance of their products will also decide the catalyst.

\section{Acknowledgements}

Some works of this study were supported by the Fund of the FY2009 Research Project of Innovative Research Organization, Saitama University, Japan. The authors also acknowledge Japanese Ministry of Education, Culture, Sports, Science and Technology (MEXT) of Japan for providing the Special Funds for Basic Researches (B) (No. 19404021, FY2007 FY2009 and No. 22404022, FY2010 2012) of Grant-in-Aid for Scientific Research.

\section{References}

[1] R. S. Lu et al., A Vital Way of Saving Wood: Utilization of China Urban Waste Wood, Wood industry, Vol, 20, 2006 (in Chinese).

[2] Wang Q. et al., Study on char-biomass briquette of pyrolyzed materials from industrial organic wastes, Proceedings of International Conference of Renewable Energy 2006, P-B-25, 1135-1140, 2006.

[3] C. Yoshida et al., Preparation of carbon fibers from biomass-based phenol formaldehyde resin, Journal of materials science, 40, 335-339, 2005. 
[4] F. Behrendt et al., Direct liquefaction of biomass, Chem. Eng. Technol. 31, 667-677, 2008.

[5] W.J. Lee et al., Novolak PF resins prepared from phenol liquefied Cryptomeria japonica and used in manufacturing moldings, Bioresource Technology, 99, 7247-7254, 2008.

[6] L.Z. Lin et al., Liquefaction mechanism of [beta]-O-4 lignin model compound in the presence of phenol under acid catalysis. Part 1. Identification of the reaction products, Holzforschung, 55, 617-624, 2001a.

[7] L.Z. Lin et al., Liquefaction mechanism of [beta]-O-4 lignin model compound in the presence of phenol under acid catalysis. Part 2. reaction behaviour and pathway, Holzforschung, 55, 625-630, 2001 b.

[8] L.Z. Lin et al., Liquefaction mechanism of lignin in the presence of phenol at elevated temperature without catalysts. I. structural characterization of the reaction products, Holzforschung, 51, 316-324, 1997a.

[9] L.Z. Lin et al., Liquefaction mechanism of lignin in the presence of phenol at elevated temperature without catalysts. II. Reaction pathway, Holzforschung, 51, 325-332, 1997 b.

[10] L.Z. Lin et al., Liquefaction mechanism of cellulose in the presence of phenol under acid catalysis, Carbohydrate Polymers, 57, 123-129, 2004.

[11] T. Yamada et al., Characterization of the products resulting from ethylene glycol liquefaction of cellulose, J Wood Sci, 47, (6), 458-464, 2001.

[12] H. Ono et al., Dissolving Behavior and Fate of Cellulose in Phenol Liquefaction, Trans. Mat. Res. Soc.Japan, 26, (3), 807-812, 2001.

[13] Y.C. Zhang et al., Characterization of liquefied product from cellulose with phenol in the presence of sulfuric acid, Bioresource Technology, 97, 313321, 2006.

[14] Lee, S.H., Ohkata, T., Rapid wood liquefaction by supercritical phenol, Wood Sci. Technol. 37, 29-38, 2003.

[15] Klok, C. and de Roos, A.M., Population level consequences of toxicological influences on individual growth and reproduction in Lumbricus rubellus (Lumbricidae, Oligochaeta), Ecotoxicology and Environmental Safety 33, pp. 118-127, 1996.

[16] M.H. Alma et al., some characterizations of hydrochloride acid catalyzed phenolated wood-based materials, Mokusai Gakkaishi, 41(8): 741-748, 1995a. 